
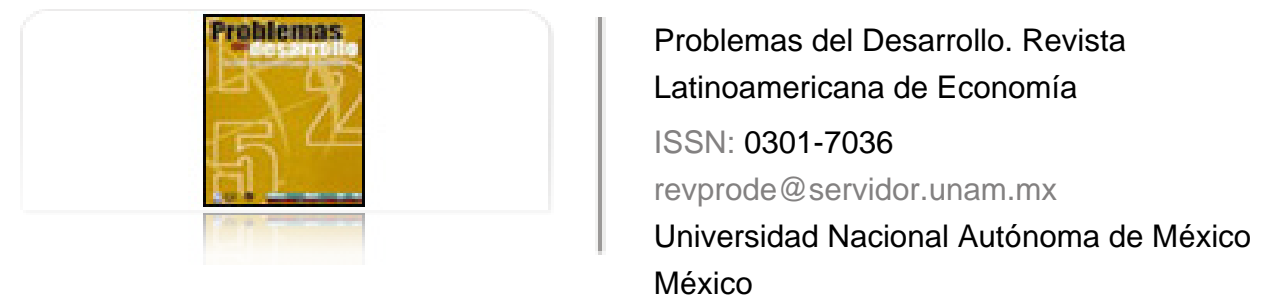

Figueroa Sepúlveda, Víctor M.

LA CRISIS GLOBAL. VIEJAS Y NUEVAS CONTRADICCIONES

Problemas del Desarrollo. Revista Latinoamericana de Economía, vol. 41, núm. 161, abril-junio, 2010, pp. 193-211

Universidad Nacional Autónoma de México

Distrito Federal, México

Disponible en: http://www.redalyc.org/articulo.oa?id=11820105008

Cómo citar el artículo

- Número completo

- Más información del artículo

Página de la revista en redalyc.org

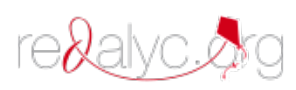

Sistema de Información Científica

Red de Revistas Científicas de América Latina, el Caribe, España y Portugal Proyecto académico sin fines de lucro, desarrollado bajo la iniciativa de acceso abierto 


\title{
LA CRISIS GLOBAL. VIEJAS Y NUEVAS CONTRADICCIONES
}

\author{
Víctor M. Figueroa Sepúlveda*
}

Fecha de recepción: 18 de septiembre de 2009. Fecha de aceptación: 5 de mayo de 2010.

\section{Resumen}

En el presente artículo se argumenta que la actual crisis es inédita en cuanto ha resultado de la operación de dos tipos distintivos de contradicciones profundas. Por un lado, aparecen los conflictos inherentes a la relación de capital. En este sentido, las causas de la presente crisis no difieren en lo fundamental de aquellas que dieron lugar a las grandes convulsiones del siglo XX. Todas las crisis informan de relaciones de clase que obstaculizan la producción porque provocan una caída de la ganancia. Por otro lado, en la actualidad el capital ha entrado en una aguda contradicción con la naturaleza, lo que condiciona la ruta que habrá de seguirse para lograr una solución duradera a los problemas que enfrenta la acumulación. En el pasado, cada nuevo periodo de crecimiento se fundó en cambios tecnológicos que apoyaron tanto la redefinición de las relaciones de clase como una mayor explotación de la naturaleza. Hoy, un diseño industrial sostenible debe considerar la protección del medio ambiente.

Palabras clave: crisis y relación de clases, estrategia neoliberal, productividad, investigación y desarrollo, cambio climático.

* Director de la Unidad de Posgrado en Ciencia Política, Universidad Autónoma de Zacatecas. Correo electrónico: vmfig2000@yahoo.com.mx 


\begin{abstract}
The author argues in this article that the present crisis is unusual in that it involves two distinct and profound contradictions. On the one hand, inherent conflicts related to capital have appeared. In this sense, the causes of the present crisis are fundamentally no different from those that gave rise to the great convulsions of the 20th century. All crises speak of class relations that obstruct production because they lead to a fall in profits. However, on the other hand, today capital has entered a sharp contradiction with nature, completely conditioning the route that will have to be followed to achieve a durable solution to the problems faced by accumulation. In the past, every new period of growth was based on technological changes that supported both the redefinition of class relations and a greater exploitation of nature. Today, a sustainable industrial design must include the protection of the environment.
\end{abstract}

Key words: crises and class relations, neoliberal strategy, productivity, research and development, climate change.

\title{
Résumé
}

Dans cet article, l'auteur soutient que la crise actuelle est inédite en cela qu'elle a découlé de la conjonction de deux types de contradictions profondes. D'une part, on peut y voir les conflits inhérents à la relation du capital. Dans ce sens, les causes de la crise présente ne diffèrent pas, fondamentalement, de celles qui ont été à l'origine des grandes convulsions du XXe siècle. Toutes les crises dévoilent des rapports de classe qui font obstacle à la production parce qu'elles sont cause d'une chute du taux de profit. Mais, d'autre part, à l'heure actuelle, le capital est entré dans une phase de contradiction aigüe avec la nature, ce qui conditionne totalement la route à suivre pour parvenir à une solution durable des problèmes auxquels fait face l'accumulation. Dans le passé, chaque nouvelle période de croissance s'est fondée sur des avancées technologiques qui ont contribué tant à la redéfinition des relations de classe qu'à une plus grande exploitation de la nature. Aujourd'hui, pour former un dessein viable pour l'industrie, il est nécessaire de prendre en compte la défense de l'environnement.

Mots clés: crise et rapport de classes, stratégie néoliberale, productivité, recherche et développement, changement climatique.

\section{Resumo}

No presente artigo, o autor argumenta que a atual crise é inédita por ser o resultado da operação de dois tipos distintivos de contradições profundas. Por um lado, aparecem os conflitos inerentes à relação de capital. Neste sentido, as causas da presente crise não diferem no fundamental daquelas que deram lugar às grandes convulsões do século XX. Todas as crises informam de relações de classe que obstaculizam a produção porque provocan uma caída do lucro. Mas, por outro lado, na atualidade o capital entrou numa aguda contradição com a natureza, o que condiciona por completo a rota que haverá de seguir-se para lograr uma solução duradeira aos problemas que a acumulação enfrenta. No passado, cada novo período de crescimiento se fundou em mudanças tecnológicas que apoiaram tanto a redefinição das relações de classe como uma maior explotação da natureza. Hoje em dia, un desenho industrial sustentável deve considerar a proteção do meio ambiente.

Palavras-chave: crise e relação de classes, estratégia neoliberal, produtividade, investigação e desenvolvimento, mudança climática. 


\section{Introducción}

1 pesar de la gran cantidad de material que se ha producido en torno a la presente crisis del capital, la discusión sobre sus causas y alcances está lejos de agotarse. Desde luego, en ciencias sociales rara vez se llegará a un consenso respecto de los aspectos fundamentales del movimiento de la sociedad, menos aún en relación con un problema tan complejo como la actual crisis. Ello no vuelve menos necesaria la discusión, pues siempre habrá un nuevo punto de vista que enriquezca el debate.

Entre las organizaciones internacionales, la UNCTAD ofrece una línea crítica de interpretación del fenómeno, que recoge tesis de gran aceptación:

1) La crisis que estalló en 2007 y se profundizó al año siguiente es resultado de la ausencia de regulaciones que controlen el movimiento de los mercados en los ámbitos nacional e internacional.

2) La plena libertad concedida a los mercados financieros y el desarrollo de las técnicas especulativas dio lugar a instrumentos financieros completamente desligados del sector productivo. Los mercados de futuro aparecen como un ejemplo sobresaliente de esta situación. El uso de los fondos en busca de ganancias no generó aumentos en el ingreso real.

3) La "incoherencia sistémica" entre los "sistemas" de comercio internacional y los de tipo financiero y monetario se tradujo en desequilibrios comerciales, endeudamiento y una desvinculación de las tasas de cambio y los diferenciales de inflación.

4) Superar la crisis requiere una reforma que permita la regulación del sistema financiero y la reorientación del crédito hacia la inversión productiva. Los mecanismos e instrumentos financieros carentes de funcionalidad social deben ser eliminados. Para llevar a cabo estos propósitos es necesaria la acción concertada de los gobiernos, con vistas a una regulación global, especialmente si se trata de controlar los mercados de futuro, evitar la especulación con divisas y el manejo de las tasas de cambio (UNCTAD, 2009).

En el discurso de la UNCTAD hay un ataque a la globalización basada en el fundamentalismo del laissez-faire y, aunque no de manera explícita, se declara el deceso de la globalización neoliberal. No obstante, el enfoque es limitado: pese a que se fundamente en la observación de procesos reales y sugiera la aplicación de medidas indispensables, se concentra en una esfera particular de la vida económica y reduce a ella los desafíos por delante. Ya el hecho de que no conceda suficiente atención a los procesos de producción, impide que devele la verdadera naturaleza de la crisis y que proponga estrategias acertadas para intentar superarla -si ello fuera posible. 
En el presente ensayo intentaremos demostrar que la crisis es integral en cuanto compromete a las diferentes esferas de la economía, en particular a la producción. Más aún, partimos de la hipótesis de que la crisis de 2007-2008 es una reedición intensificada de las convulsiones que tuvieron lugar en 2000-2002, y es una clara evidencia de que el capitalismo está entrampado en contradicciones internas que no ha logrado resolver. Desde una perspectiva más amplia, se mostrará que las dificultades que ha enfrentado la economía estadounidense durante el periodo neoliberal están vinculadas a la estrategia seguida para superar los trastornos de finales de la década de 1960 y principios de la de 1970.

Sin embargo, para dar cuenta de esta crisis en particular, este enfoque debe ser complementado. En la actualidad, el capital no sólo se encuentra en contradicción consigo mismo, sino también está en un grave conflicto con la naturaleza. Este conflicto levanta restricciones tanto a la búsqueda de ganancias como a la ruta tecnológica que habrá de orientar el desarrollo de las fuerzas productivas.

Iniciaremos con una breve presentación de los principales postulados teóricos del enfoque que hemos adoptado. Enseguida describiremos los datos más sobresalientes de la "economía real" y de los movimientos en la esfera financiera. El tercer apartado está dedicado a algunas reflexiones sobre la situación del medio ambiente y la crisis energética. Al final, se esbozan las conclusiones más importantes.

\section{La crisis. Puntos teóricos clave}

Las crisis se producen debido a que la tasa de ganancia cae y desalienta la inversión. Un postulado fundamental del marxismo es que esta caída es tendencial y ocurre, con recuperaciones periódicas, durante el largo plazo del capitalismo, debido a un incremento en la composición del capital. Como tal, esta tendencia da cuenta de la historicidad del modo capitalista, pero no explica las grandes crisis periódicas ni las recuperaciones consiguientes. La caída como tendencia del desarrollo capitalista debe ser diferenciada de la caída periódica concreta dentro del sistema. Según lo vemos, la explicación de esta última contiene los siguientes momentos teóricos:

a) la tasa de ganancia cae porque cae el plusvalor, o porque éste no aumenta de manera suficiente para contener la caída;

b) ello ocurre porque la posición económica del trabajo respecto del capital ha mejorado: la capacidad del capital para extraer plusvalor se ha debilitado;

c) el incremento del grado de explotación y del nivel de la tasa de ganancia requiere, como condición previa, la modificación de la correlación de clases que produjo el estancamiento; 
d) esta redefinición de la relación de clases es el resultado de procesos que toman lugar en tres áreas distintas, pero complementarias, del proceso social: 1) por un lado, tenemos la acción espontánea de la crisis, al crear desempleo, agudizar la competencia entre trabajadores y reducir los niveles salariales; 2) por el otro, la acción del Estado tendiente a sostener y aun a profundizar la debilidad del trabajo, al menos mientras la tasa de ganancia se recupera, y 3 ) desde que el trabajo se fortaleció en el marco de una determinada relación técnica de la producción, la consolidación de nuevos niveles de productividad y de intensidad del trabajo, esto es, de nuevos y más elevados niveles de explotación, necesita crear un nuevo modo técnico de producir, mediante la acción combinada del Estado y los empresarios para crear progreso tecnológico. Lo que sigue a la crisis es, pues, una ola de innovaciones tecnológicas, un rediseño de la producción industrial, junto con la desvalorización de capitales inadecuados, o la prolongación de su utilidad en otros lugares.

La Gran Depresión de la década de 1930 en los Estados Unidos dio cuenta de la operación de todos los procesos indicados:

a) un drástico proceso de deterioro de las posiciones del trabajo, con niveles de desempleo que se elevaron considerablemente, generando hambre, pobreza y desánimo popular, en suma, un envilecimiento general de las condiciones de vida del pueblo;

b) una destrucción masiva de capitales que se profundizó con la guerra;

c) una renovación técnica que extendió la explotación de recursos fundamentales ya existentes como el petróleo, la electricidad y el acero, junto con una mejor organización de la ciencia con aplicación productiva, la cual, en gran medida, tomó ventajas de sus propios avances en las tecnologías militares;

d) el desarrollo de nuevas formas de organización del trabajo, en particular el fordismo;

e) el envío de industrias obsoletas hacia los países subdesarrollados;

f) la continuación de la represión al movimiento obrero tras la guerra, junto con concesiones que luego consagrarían un nuevo pacto social, a partir del cual el trabajo iniciaría su propia recuperación.

La crisis que estalló a finales de la década de 1960, y que tuvo manifestaciones drásticas ya iniciados los setenta, da cuenta de reacciones similares. Tras el desempleo y la caída de los salarios, se introdujo la flexibilización laboral y el modo técnico del capitalismo fue objeto de modificaciones, con la revolución en la microelectrónica, la 
informática, la automatización, las redes de computación, aunque estas transformaciones tendieron a concentrarse en la industria de las tecnologías de la información y la comunicación (TIC) y de los servicios. La ciencia produjo avances también en otros campos, especialmente en la biogenética y la bioquímica. La organización laboral también adoptó formas más adecuadas a la nueva realidad técnica. Sin embargo, el capitalismo no logró dar lugar a una etapa de crecimiento relativamente elevado y sostenido, por razones que señalaremos más adelante. Aquí nos concentramos en el caso de los Estados Unidos, porque es el centro de las convulsiones globales.

\section{La estrategia estadounidense}

La estrategia neoliberal, conocida como globalización, buscó articular tres factores: a) la flexibilización laboral, $b$ ) el librecambio y c) la financiarización. Este último proceso, en particular, daría cuenta del predominio del capital financiero en sentido restringido (bancos, bolsas, seguros; en sentido amplio, este concepto incluye al capital industrial), reflejándose en un incremento de la participación de los intereses sobre las ganancias productivas y una transferencia de ingresos de la "economía real" hacia el sector financiero. El método principal de la financiarización es el endeudamiento (Palley, 2007).

El crecimiento económico, con una tendencia a la caída en el largo plazo (Palley, 2007), fue errático. El PIB creció entre 1987 y 1989 a tasas superiores al 3.4\%, descendió en 1990 y 1991, se recuperó entre 1992 y 1994 (3.3\% y 4.0\%, respectivamente), disminuyó en 1995 (2.5\%), y entre 1996 y 2000 se mantuvo a una tasa ligeramente superior a 4\%, aunque entre 1999 y 2000 cayó de $4.5 \%$ a 3.7\%, cifras que dan cuenta de una crisis en proceso.

La flexibilización laboral (que incluye el debilitamiento de los sindicatos, el recurso a la subcontratación, la reducción de los apoyos a los ingresos laborales en la forma de beneficios por desempleo y otros y, en general, el desmantelamiento de derechos laborales) fue concebida como un mecanismo orientado a estabilizar la situación de debilidad del trabajo y el papel dominante del capital. No se trataba simplemente de profundizar los efectos de la crisis provocados por la introducción del neoliberalismo. Debía permitir la transferencia continua de recursos, con vistas a sostener las ganancias, al desvincular los ingresos del trabajo de los aumentos de productividad. El salario relativo (respecto del plusvalor) debía caer de manera sostenida.

Desde una perspectiva de largo plazo, este mecanismo funcionó perfectamente. La distribución del ingreso se deterioró de manera consistente a partir de 1980. En 
ese año, el quintil más bajo recibía 5.3\% del ingreso, y el quintil más alto, 41.1\%. En 2007 , el primero participaba con $4 \%$, y el segundo, con $48.5 \%$. El $5 \%$ más rico elevó su participación de $14.6 \%$ a $21.8 \%$ en ese periodo (US Census Bureau, 2008). Sin embargo, esta tendencia es contenida por periodos. Entre 1994 y 2000, la participación del quintil más bajo mejoró ligeramente, al pasar de $4.1 \%$ a 4.3 por ciento.

Una tendencia similar se registra para la participación de los salarios en el ingreso. Según cálculos de Buchele y Christiansen (2007), que no coinciden con otras fuentes excepto en relación con la tendencia general, la cuota del trabajo en el ingreso que a principios de la década de 1970 era de $71 \%$, cayó en 10 puntos porcentuales en 2005. Estas cifras se logran sustrayendo los ingresos del $0.5 \%$ de los salarios más altos, en el entendido de que se trata de ingresos que reciben los ejecutivos que actúan representando a los propietarios. Según los autores, este sector incrementó su participación de $3 \%$ a $9 \%$ en ese periodo.

En 1997, el costo de la unidad de trabajo en el valor agregado real bruto del sector no financiero comenzó a elevarse de manera sostenida hasta el año 2001, mientras que la unidad de ganancia caía de la misma manera en ese periodo (BEA, 2009b). La relación entre productividad y salarios se modificó: "las remuneraciones reales tuvieron una recuperación; aumentaron a un ritmo ligeramente superior al de la productividad" (CCL, 2003: 156), a pesar de que la productividad mantuvo su crecimiento después de 1996.

En otras palabras, se desactivó el mecanismo por el cual el capital no financiero estadounidense estaba obteniendo ganancias y estímulos para el crecimiento. De esa manera se desmoronaba uno de los puntales de la estrategia neoliberal. Independientemente de su debilidad subjetiva en términos de organización, el trabajo logró ganar posiciones gracias a los cambios en su situación objetiva. En efecto, a partir de 1994 la tasa de desempleo empezó a caer de manera consistente. En enero de ese año fue de $6.6 \%$ y en el mismo mes de 2000 se redujo a $4 \%$; en los últimos cuatro meses de este año se mantuvo en $3.9 \%$, con lo que la economía prácticamente volvió a una situación de pleno empleo (BLS, 2009).

Esta situación no ofrece muchos créditos a los cambios en la productividad del trabajo, puesto que el crecimiento logrado en esos años no fue capaz de contener el crecimiento del empleo. Y, de hecho, la evolución de la productividad laboral, según datos de la OCDE, no es tan deslumbrante. Después de 1995, año para el cual se reporta un crecimiento $=0$, evolucionó de la siguiente manera: 1996: $2.5 \%$; 1997 : 1.5\%; 1998: 2.0\%; 1999: 2.5\%; 2000: 2.3\%. El sector económico que contribuyó en mayor medida al crecimiento de la productividad en este periodo, fue el de las TIC, 
tanto por el crecimiento que aportaba directamente, en particular en el campo de las computadoras, como por su impacto en la organización de los negocios en el resto de los sectores. Para apreciar su contribución como sector, Susan Houseman (2006) llama la atención sobre el hecho de que mientras el producto de una hora de trabajo en toda la manufactura creció $45 \%$ de 1990 a 2000, en computadoras y productos electrónicos lo hizo un $426 \%$. La autora también destaca que entre los factores que incidieron en el crecimiento del sector es necesario incluir los ahorros logrados en fuerza de trabajo tanto por medio del outsourcing como del offshoring.

La inversión en TIC creció poderosamente, muy por encima del promedio del total de la inversión privada fija, estimulando su difusión, apoyada por la especulación que, a su vez, terminaría empujando los precios artificialmente al alza, pese a los avances en productividad.

Los cálculos sobre el impacto de la industria de la tecnología de la información difieren, pero el consenso al respecto es predominante. Las formas en que las tecnologías de la información pueden impactar sobre la productividad son muchas y variadas e incluyen desde el diseño de productos hasta su comercialización (Stiroh, 2002). También se destaca el papel que la innovación en la organización laboral tiene sobre el incremento de la productividad (Lynch, 2007). Sin embargo, en el resto de las industrias estas tecnologías no modifican por sí mismas la relación directa entre el trabajador y sus medios de producción (excepto en algunos casos, como el estímulo a la introducción de robots), es decir, no dan cuenta de un verdadero avance de las fuerzas productivas en ese plano. Por otro lado, las industrias beneficiadas por la introducción de esas tecnologías son las que operan con los procesos más modernos. La enorme diferencia entre el crecimiento de las TIC y el del resto de la industria son compatibles con estos dos fenómenos. Pero no explican la estrategia de desarrollo industrial estadounidense.

En realidad, era imposible impedir que los niveles de empleo aumentaran si no se garantizaba el desplazamiento de fuerza laboral por medio del desarrollo técnico de la producción. En la estrategia neoliberal, a este aspecto no se le concedía la debida importancia. Como ya se ha reconocido, el impulso al desarrollo del conocimiento y a sus aplicaciones productivas es crucial para el desarrollo económico en general y el de la productividad en particular. El gobierno de Ronald Reagan introdujo un cambio radical en este terreno. El gasto en investigación y desarrollo (ID) para la industria se redujo de $1.5 \%$ del gasto total en 1976 a $0.4 \%$ en 1982, es decir, cayó prácticamente a una cuarta parte. También disminuyó el porcentaje del gasto total destinado a la investigación en energía: de 9.6 a 6.7. Lo mismo pasó con la investigación sobre medio 
ambiente. En cambio, el gasto dedicado a defensa creció: de $49.6 \%$ a $64.3 \%$ del gasto total en el mismo periodo. El país retrocedió en su gestión estatal del desarrollo, el crecimiento de la productividad perdió fuerza, la economía debilitó su competitividad y su posición en el mercado mundial se deterioró. El capitalismo estadounidense puso en riesgo su posición dominante en la producción de progreso, y de hecho fue desplazado en muchas ramas de la economía (Figueroa, 1992, 1993).

Esta situación se mantuvo, con pocas modificaciones relevantes, durante las últimas dos décadas del siglo pasado. La investigación en tecnología industrial recibió sólo el 0.2\% del gasto total en ID en 1990, subió a 0.3\% entre 1991 y 1994 y alcanzó alrededor de $0.6 \%$ entre 1995 y 2000, todavía apenas sobre un tercio de su participación en 1976. El gasto en ID en energía continuó cayendo: de $4.5 \%$ en 1991 a 1.5\% en 2000. A pesar de que la inversión para investigación en defensa cayó paulatinamente hasta 53.2\% en el año 2000, se mantuvo por arriba de los niveles de 1976. La investigación en salud fue la principal beneficiaria de esta nueva redistribución del gasto en ID, con un 20.9\% del gasto total en 2000 (RICYT, 2009).

Bajo la conducción neoliberal, el país retrocedió en sus esfuerzos por impulsar el desarrollo industrial. El proyecto de obtener plusvalor mediante el deterioro de los salarios justificaba esta conducta. Sin embargo, por esa misma razón, no podía ofrecer una salida integral, con niveles de crecimiento relativamente elevados y sostenidos, a la crisis de fines de los sesenta y principios de los setenta.

Pero no renunciaba al crecimiento económico. Para impulsarlo, aparte de la flexibilización laboral, optó por "globalizar" sus fuentes de provisión de medios para la industria. Adoptó el librecambio como la consigna principal en sus relaciones con el resto de los países. El supuesto que animaba esta política era que las firmas involucradas en el comercio internacional son más productivas, crean más empleo y pagan mejores salarios. Esta política también ocasionaba problemas a las industrias que carecían de ventajas competitivas, pero ésas eran consideradas dificultades de corto plazo: la competencia las forzaría a elevar sus niveles de competitividad. El desarrollo industrial sería un problema que las firmas prácticamente podrían atender por sí mismas, como resultado de los estímulos provistos por el mercado.

Ya en 1979, la Buy American Act, aprobada en 1933 para impulsar el consumo de bienes producidos internamente, fue reemplazada por la Trade Agreements Act, que amplía decisivamente el campo de productos que pueden adquirirse en el exterior. Con vistas a beneficiar a la industria estadounidense, el gobierno puso especial atención en la tarea de derribar las barreras a la inversión extranjera en el resto de los países, principalmente en los subdesarrollados y los "emergentes", sobre todo 
luego de que se desplomaron los obstáculos representados por la Unión Soviética. Ello permitiría -y lo hizo- beneficiarse de la explotación de fuerza de trabajo barata en el exterior, tanto a través del offshoring como de la importación en general. De esa manera también fue posible reducir costos y precios, incrementar la producción y mostrar un trabajo artificialmente más productivo. En particular, esta estrategia benefició a las TIC y estuvo acompañada de la internacionalización de la ID, que si bien haría más lentos los procesos de innovación, también implicaría una reducción en los costos, gracias a una fuerza de trabajo cuyos precios compensarían con creces las pérdidas causadas por sus menores competencias (Houseman, 2006). En suma, la globalización extendía hacia el exterior el proyecto de obtener ganancias descansando en una fuerza laboral barata.

La estrategia estadounidense, al renunciar parcialmente al desarrollo de las fuerzas productivas, abrió la posibilidad a otros países de elevar su participación en esta tarea. Pero ello implicaba costos. El déficit comercial, como cabía esperar, continuó creciendo. De 1990 a 2000 pasó de US \$80 864 millones a US \$379 835 millones, es decir, aumentó 4.7 veces. El excedente en el comercio de servicios que era de US \$30 173 millones en el primer año, alcanzó su punto más alto en 1997, cuando empezó a caer para alcanzar US \$74 855 millones en 2000, y apenas amortiguar el efecto del déficit de bienes que alcanzó US\$ 454690 millones el mismo año (BEA, 2009c). El sobreconsumo estadounidense, o sea, la diferencia entre el consumo tanto personal como productivo y la producción al interior de la economía, debía cubrirse mediante el financiamiento externo.

La ventaja de un Estado que puede endeudarse en su propia moneda, que es al mismo tiempo la principal reserva mundial, lo que informa del seignorage monetario internacional del país, contribuyó decisivamente al financiamiento del crecimiento económico. En efecto, tanto gobiernos como particulares extranjeros tuvieron acceso a una gran variedad de bienes, privados y públicos, que incluían valores, bonos, títulos del Tesoro, bienes inmobiliarios y empresas estadounidenses, todos denominados en dólares. Una gran parte de los activos estadounidenses se desnacionalizaron, pero ello sólo representaba ventajas, puesto que la inversión extranjera estimulaba el crecimiento y creaba puestos de trabajo. De este modo, la inversión doméstica podía crecer por encima del ahorro interno. Al mismo tiempo, el gobierno incrementaba su deuda, pero ello era necesario mientras la reorganización interna hiciera su proceso. Además, por estos medios, los bienes internos aumentaban sus precios, reforzando su papel de fuente de ingreso, mientras permitían sostener bajas tasas de interés. 
Desde Japón y China llegaron la mayor parte de los fondos. Estos intercambios eran promovidos por intereses comunes. Por ejemplo, China se veía estimulada a colocar parte de su excedente comercial en los Estados Unidos, con la perspectiva de proteger su propia moneda, en el entendido de que los países necesitan depositar sus fondos en el país emisor de reservas, puesto que requieren de éstas para realizar sus acuerdos comerciales.

Al mismo tiempo, el déficit comercial implicaba otras consecuencias. Por un lado, trasladaba a otros países la producción de bienes internamente necesarios, en la medida en que desplazaba hacia estos últimos inversión productiva y promoción del empleo. Por el otro, las inversiones en los Estados Unidos de los países con excedentes comerciales normalmente no se dirigirían a las industrias que, en ese país, representaran una competencia para ellos. También, si proveían créditos para compras estadounidenses en sus países, esos créditos irían orientados a reforzar su propia capacidad de exportación. Todo lo anterior debía deteriorar la producción manufacturera de los Estados Unidos. Y también, finalmente, empujaba a la especialización productiva, que, en este caso, se orientó hacia los servicios y las industrias de tecnologías de la informática.

No se puede decir que esto último fuera un resultado colateral de la estrategia estadounidense. Aun en el Economic Report of the President (ERP) de 2007, se puede apreciar esta confianza en el librecambio y en las bondades de la especialización. En efecto, allí se afirma: “...los Estados Unidos pueden producir muchos servicios a un costo más bajo que nuestros socios comerciales y ellos pueden producir otro conjunto de bienes y servicios a un costo menor que los Estados Unidos" (ERP, 2007: 171). Desde luego, esta postura está basada en la convicción de que los servicios, en particular aquéllos basados en el conocimiento y en las tecnologías de la información, constituyen la fuerza económica del presente y de que los Estados Unidos han desarrollado una superioridad en este campo (ERP, 2007: 171-173).

Volviendo a la relación doméstica entre el capital y el trabajo, era probable que los cambios que hemos descrito se tradujeran en una caída de la tasa de ganancia. Dumenil y Lévy (2004), con su propia metodología, detectaron que esta evolución tomó lugar a partir de 1997-1998, en las corporaciones no financieras. Datos del BEA indican que la masa de ganancia de las corporaciones no financieras, un dato frente al cual el gran capital es mucho más sensible, cayó de 544.1 billion dollars a 487.5 billions entre 1998 y 2000. En general, si cae la masa de ganancia, caerá su tasa, a menos que la posición del trabajo se deteriore drásticamente, lo que no ocurrió en la segunda mitad de la década de 1990. 
La crisis estaba en proceso. La inversión doméstica bruta privada redujo su crecimiento de $12.4 \%$ en 1997 a $5.7 \%$ en 2000, hasta desplomarse a $-7.1 \%$ en 2001, para volver a retroceder a $-2.6 \%$ en 2002. La inversión extranjera directa tuvo una caída estrepitosa en 2001. Como era de esperar, el PIB apenas avanzó $0.8 \%$ en 2001 y $1.6 \%$ en 2002. El desempleo volvió a aumentar, alcanzando $5.7 \%$ en 2001. Los desembolsos salariales también cayeron, especialmente en la industria privada productora de bienes. El trabajo perdía claramente muchas de las posiciones conquistadas en el último lustro del siglo pasado.

Una salida duradera a la crisis demandaba una modificación profunda del modo técnico de producir, que permitiera aumentos autogenerados en la productividad y en la intensidad del trabajo, que consolidara nuevos niveles de desempleo y que mejorara la posición de la economía en el mercado mundial. Sin embargo, la conducción económica y política del país optó por reforzar la estrategia que se venía poniendo en marcha, ahora con mayor énfasis en el endeudamiento de las familias y las empresas, a fin de hacer crecer el mercado interno. En el plano de las opciones disponibles, el país, que había relajado sus esfuerzos por impulsar el desarrollo de las fuerzas productivas, no estaba preparado para otra cosa en lo inmediato y la misma conducción política del país tampoco estaba ideológicamente predispuesta a ello.

Los resultados han sido ampliamente discutidos. La productividad del trabajo creció alrededor de $2.8 \%$ anual entre 2002 y 2004 , y a partir de entonces la tasa de crecimiento cayó a 1.4\% en 2005 y $0.9 \%$ en 2006, recuperándose levemente en 2007 (1.3\%) (OECD, 2009). El desempleo cayó a 4.5\% en 2006. Los trabajadores mejoraron su posición en el ingreso. Sus remuneraciones crecieron por arriba del crecimiento de las ganancias corporativas. Su participación en el ingreso nacional pasó de $63.9 \%$ a $64.4 \%$ entre 2006 y 2007, y aunque todavía no alcanzaba el nivel de 2001 (66.2\%), tendía a mejorar. El déficit comercial continuó creciendo hasta duplicarse entre 2000 y 2006. Los precios del petróleo se incrementaron notablemente, en parte estimulados por la especulación, mientras que los precios de las exportaciones chinas aumentaban entre 2006 y 2007, como resultado de los cambios en sus propios salarios y una cierta reevaluación del yuan. La inversión fija privada doméstica, que apenas había crecido 1.9\% en 2006, se derrumbó hasta -3.1\% en 2007 (BEA, 2009a). Una nueva oleada de estancamiento económico, desempleo y caída de los salarios reeditó los males de 2001-2002 en un nivel más profundo.

El endeudamiento de los hogares y de las empresas buscaba estimular el consumo con cargo a la creación futura de valor y de ese modo impactar sobre la producción presente. En parte, el mecanismo fue exitoso por un periodo, como puede apreciarse 
en el boom inmobiliario. Pero generó precios y capitales ficticios que se desenvolvían sin contacto con la producción real, en correspondencia con el tipo de demanda que el esquema creaba. Una vez que el derrumbe del capital no financiero desmoronó las expectativas de ingresos futuros, el mundo artificialmente creado estalló en pedazos, a partir de la ruptura de su eslabón más débil: los créditos precarios (subprime), y puso en evidencia el enorme costo de una especulación que también afectaba los precios de materias primas y alimentos, elevando costos productivos e inhibiendo la producción.

La crisis de 2000-2002, en los hechos, fue manejada como si se tratara de la explosión de una burbuja, en este caso la llamada dot-com bubble que estalló en marzo de 2000 y que, a su vez, fue interpretada como el resultado de las elevadas tasas de interés y de las cargas tributarias, y no como el efecto de una caída en la demanda creada por la inversión decreciente en los sectores que no eran objeto de la especulación. Los sectores productivos que no estaban en el centro del avance tecnológico, ya no podían seguir sosteniendo la expansión de la "nueva economía". Sin embargo, desde el punto de vista dominante, para superar el estancamiento no se requería mucho más que bajar las tasas de interés (la reducción fue sistemática desde enero de 2001 hasta llegar a $1 \%$ en junio de 2003), recuperar el financiamiento externo y abrir paso a la operación de cuanto mecanismo financiero permitiera lanzar dinero al mercado. Ninguno de los problemas estructurales fue atacado, y éstos no tardaron en aflorar.

A mediados de 2009, las dos primeras estrategias mencionadas (bajas tasas de interés y financiamiento interno y externo) aún están en uso en los Estados Unidos; la tercera busca corregirse, mediante la introducción de esquemas regulatorios. Por otro lado, se está aumentando el gasto en investigación y desarrollo, pero todavía no se vislumbra una nueva oleada de cambio tecnológico. En sentido estricto, esto último impide la adopción de una estrategia más adecuada para superar la crisis. El capitalismo estadounidense, por tanto, más allá de una eventual recuperación transitoria, que postergaría las transformaciones necesarias, continúa siendo muy vulnerable.

\section{La contradicción entre el capital y la naturaleza}

Los trabajos del Grupo Intergubernamental de Expertos sobre el Cambio Climático (IPCC, según sus siglas en inglés) han acumulado evidencia que demuestra:

1) el aumento de las temperaturas, llamando la atención sobre el hecho de que 11 de los 12 años que comprenden el periodo 1995-2006, figuran entre los más cálidos desde 1850;

2) la elevación del nivel del mar, que se ha agravado entre 1993 y 2003; 
3) la reducción de las extensiones de hielo y nieve;

4) el incremento de las precipitaciones en vastas zonas del planeta y probablemente (sobre 66\% de probabilidades) de las extensiones afectadas por las sequías en todo el planeta;

5) la mayor actividad ciclónica tropical en el Atlántico Norte aproximadamente desde 1970.

El informe del IPCC sostiene que las concentraciones atmosféricas de dióxido de carbono $\left(\mathrm{CO}_{2}\right)$, metano $\left(\mathrm{CH}_{4}\right)$ y óxido nitroso $\left(\mathrm{N}_{2} \mathrm{O}\right)$, que son gases de efecto invernadero, han aumentado por efecto de las "actividades humanas". Las emisiones de $\mathrm{CO}_{2}$, el más importante, aumentaron 80\% entre 1970 y 2004 (IPCC, 2007).

Numerosas investigaciones y reportajes dan cuenta de lluvias torrenciales que han paralizado por horas a ciudades completas o provocan gran cantidad de damnificados, infecciones y muertes; olas polares que matan decenas de miles de animales; incendios forestales que destruyen miles de hectáreas; expansión de zonas pobres en oxígeno; expulsión de especies de sus entornos naturales; desplazamientos de poblaciones y muchas otras calamidades que han contribuido a formar una conciencia generalizada sobre esta realidad.

Las investigaciones sobre los efectos económicos globales del cambio climático han agudizado las preocupaciones al respecto. En particular, el Informe Stern -dirigido por Sir Nicholas Stern, jefe de Servicios Económicos del Gobierno, por encargo (en julio de 2005) del primer ministro británico, y publicado el 30 de octubre de 2006-, debía encender focos de alarma que ya no podían ser ignorados. Los puntos más destacados de este reporte son los siguientes:

1) Es necesario actuar de manera urgente para combatir los efectos del cambio climático. Ya no es posible detener el cambio climático que se producirá en las dos o tres décadas que siguen, pero se puede reducir su impacto. Estabilizar la situación en cualquier nivel requiere que las emisiones anuales se reduzcan en más del $80 \%$ de su nivel actual.

2) No hacerlo podría ocasionar la pérdida, como mínimo, de 5\% del PIB anual global. Las pérdidas podrían llegar al 20\%, o más.

3) Se requiere destinar, desde ahora, el 1\% del PIB global anual. Estos recursos podrían ser insuficientes si la innovación orientada a producir tecnologías bajas en carbono fuera más lenta de lo esperado. La inversión en ID en energía debe duplicarse, y el apoyo a la difusión de tecnologías bajas en carbono, multiplicarse por cinco. 
4) Sin lugar a dudas, la falta de acción tendrá costos mucho mayores que los destacados en el punto anterior. Además, se espera que la creación de tecnologías, mercancías y servicios bajos en carbono abran nuevas fuentes productivas ventajosas para los negocios. El desarrollo económico se puede lograr sin eludir el problema del cambio climático.

5) La reducción de las emisiones exige el mejoramiento de la eficiencia energética, la introducción de cambios en la demanda y de tecnologías limpias en los sectores de la energía, la calefacción y el transporte. Aun así, es posible que todavía en el año 2050 los combustibles fósiles sigan representando más del 50\% del suministro mundial de energía. El carbón seguirá siendo una parte importante de la mezcla energética, por lo que es indispensable promover la captura y el almacenamiento del carbono. Igualmente es necesario reducir las emisiones que resultan de la deforestación y de los procesos agrícolas e industriales.

6) La acción del Estado es imprescindible en un gran variedad de tareas para enfrentar el cambio climático. El Informe destaca la regulación del precio del carbono, el estímulo a la innovación tecnológica, la promoción de la eficiencia energética y la concertación internacional, condición necesaria para enfrentar los desafíos.

De acuerdo con el Informe: "El cambio climático es el mayor fracaso del mercado jamás visto en el mundo..." Esta sentencia puede leerse como un grito desesperado por poner a la razón al mando de la conducción económica del mundo; pero el Informe no se declara contra la lógica capitalista que, determinada por el afán de ganancia, empujó a una relación irracional con la naturaleza, aunque sí condena el liberalismo a ultranza que ha dominado los asuntos del mundo en las últimas décadas.

Resulta difícil imaginar que se pueda producir algo sin incrementar los costos mientras se avanza hacia la estabilización del sistema climático. Bastaría pensar en los aumentos de los precios del agua o de la electricidad, incluso sin considerar los precios agrícolas, del suelo, de las residencias, de los seguros, etc., para tomar nota de la magnitud de los efectos previsibles.

La toma de conciencia generalizada respecto del cambio climático y de la urgencia de enfrentarlo ha sido reforzada por la observación del desenvolvimiento del petróleo, un recurso natural que no puede reproducirse en los términos requeridos por el crecimiento económico. Se trata del recurso natural que mayor impacto ha tenido en el crecimiento y el desarrollo económicos durante el siglo XX y lo que va del actual. La convicción es que la mayor parte de los yacimientos "fáciles" en 
el mundo han alcanzado su cenit (pico o meseta, según los casos), y que a partir de ahora la producción deberá concentrarse en los yacimientos de explotación más difícil y, por lo mismo, demandará inversiones y costos más elevados. El petróleo no convencional, aparte de ser más contaminante, requiere más agua y energía para su procesamiento.

Los precios del petróleo están también determinados por la demanda, lo cual explica sus oscilaciones. El bajo crecimiento económico favorece precios relativamente bajos, pero ello, por un lado, lleva a la postergación de inversiones en yacimientos de explotación más difícil y, por otro, daña de manera adicional el desenvolvimiento económico de los países exportadores. Las señales de crecimiento económico tienen el impacto inverso.

No parece razonable descartar nuevos descubrimientos de petróleo convencional, como ya ocurrió en Alaska (lo que de todos modos mantiene a los Estados Unidos por debajo de su pico de 1970) y en Rusia (que sí superó su pico), pero no ha habido mayores noticias al respecto. De hecho, la tasa de descubrimientos de yacimientos petroleros empezó a caer desde la década de 1960, y a partir de 1981 el consumo de petróleo ha crecido con más rapidez que la tasa de dichos descubrimientos.

El petróleo convencional, por su impacto en la producción y sus bajos costos, las facilidades para almacenarlo y transportarlo, vino a significar algo así como un bono energético que hizo posible toda una época de expansión capitalista, más allá de sus inevitables contracciones periódicas. La evidencia del cenit de este recurso natural en la mayor parte de los países productores está anunciando el fin de esa época. Cuándo tomará lugar este evento a escala de la producción mundial es objeto de estimaciones distintas en el seno de la comunidad científica dedicada al estudio del peak oil, tema que, aun cuando está condicionado por consideraciones económicas y políticas, cae finalmente en el campo de la geofísica.

La búsqueda de alternativas energéticas ha dado lugar a nuevas contradicciones. Es el caso de la agricultura. La producción de bioenergéticos se ha logrado en parte mediante el desplazamiento de la producción de alimentos, de por sí afectada por el cambio climático. La crisis alimentaria no es el resultado de la dinámica propia de la agricultura, sino de influencias, hasta cierto punto, externas a la misma. Y señalamos hasta cierto punto, porque el crecimiento agrícola ha sido posible gracias al concurso del petróleo, a través de los agroquímicos, los sistemas de refrigeración, la movilización de tractores y tráilers, además del recurso de los fertilizantes que provienen del gas natural, así como a la electricidad de plantas termoeléctricas que producen calor a partir de combustibles fósiles. Es decir, el sistema agrícola también es un emisor de 
gases de efecto invernadero, y, en la medida en que contribuye al cambio climático, también da lugar a las causas que lo afectan (inundaciones, sequías, plagas, erosión de la tierra). Pero no parece razonable pensar que la presente crisis alimentaria hubiera alcanzado en tan poco tiempo sus manifestaciones actuales sin la intervención de la producción de bioenergéticos. Así, es también un efecto de la respuesta capitalista al problema energético.

\section{Para concluir}

El mundo está ante una nueva ola de cambios tecnológicos. Para satisfacer los intereses capitalistas, esta nueva ola debe producir una nueva relación de la producción con el trabajo, y con la naturaleza. En el pasado, la superación de las crisis tuvo lugar, indefectiblemente, mediante un salto en la explotación del trabajo y también de la naturaleza. Los nuevos y más poderosos medios de producción afectaban a ambos. Ahora es urgente proteger a la naturaleza. Ésta es la magnitud del desafío que el capitalismo tiene por delante.

El descubrimiento de una fuente energética limpia, fácilmente disponible y barata sería un gran paso adelante en ese sentido. Tal condición no existe, al menos por ahora. Es generalmente reconocido que no sólo no existen alternativas, sino tampoco alguna combinación de opciones que se aproxime a la densidad energética del petróleo. El petróleo ya no ofrece promesas viables, no sólo por sus emisiones de dióxido de carbono, sino también porque se está agotando como recurso barato. Sin embargo, rediseñar la industria es por sí mismo costoso, y los estímulos para el cambio se reducen aún más si han de tener lugar en el marco de una expectativa de ganancias a la baja. Por lo demás, el capitalismo estadounidense poco o nada se esforzó por preparar una innovación masiva en el campo de la industria y la energía, como puede apreciarse en el bajo estímulo a la investigación en estas áreas. Más bien dejó sujeto a su superioridad bélica el control de las fuentes de energía. El capital no está preparado para afrontar una recuperación de sus niveles de ganancia por medio del progreso tecnológico. Tampoco lo está para operar con menores niveles de ganancias, como lo han evidenciado los estancamientos de la producción en los últimos años.

Cabe esperar, por tanto, que seguirá recurriéndose a los recursos existentes. Esta ruta puede ser reforzada con el descubrimiento de métodos que hagan posible un enfriamiento del planeta y permitan extender la captura y almacenamiento de las emisiones. En este contexto, el aumento de los costos en capital constante deberá compensarse con un abaratamiento proporcional de la fuerza de trabajo y una exten- 
sión de la pobreza. En lo inmediato, el gobierno estadounidense se ha concentrado en el rescate y ordenamiento financieros, mientras una vez más deja la reactivación económica en manos de la financiarización de la economía. Se puede, entonces, esperar una leve mejoría económica, seguida por una nueva y más profunda recesión. Mientras no se dé lugar a una revolución tecnológica orientada a la explotación de nuevas energías, el empeño de conciliar la ganancia con la naturaleza, los intereses del capital con los intereses del planeta, se probará cada vez más inútil.

La competencia por los recursos existentes se agravará. La tendencia al enfrentamiento bélico entre potencias puede ser controlada, al menos parcialmente, mediante la introducción de una multilateralidad que coordine a los grandes poderes en la conducción de los asuntos globales, así sea por un determinado periodo. Ello demandará ciertas concesiones por parte de los Estados Unidos; al parecer, ya se están preparando para ello, en cuanto a una redefinición de las zonas de influencia y control por parte de las viejas y nuevas potencias. Seguramente, las resistencias de ese país serán mayores en relación con las demandas actuales para crear un nuevo orden monetario internacional, en particular si de lo que se trata es actualizar la iniciativa de Keynes de crear una moneda especial de reserva. Los Estados Unidos no estarán dispuestos a renunciar a las ventajas de su actual posición privilegiada. Pero al mismo tiempo, aunque el orden monetario vigente es visto como una de las causas principales del actual estado de cosas, potencias como China, interesadas en la reforma, no logran todavía encontrar una fórmula que no termine lesionándolas a ellas mismas. Esto fortalece las resistencias estadounidenses.

En cuanto a la renegociación de las zonas de influencia en el mundo, no se vislumbran cambios para América Latina. A las medidas de control a través de los tratados de libre comercio, se agrega ahora el uso de siete bases militares colombianas por los Estados Unidos, incluyendo el establecimiento de una base de operaciones de la Fuerza Aérea y la reactivación de la Cuarta Flota. El surgimiento de gobiernos populares y su posible extensión a otros países debe anotarse como la principal causa de estas medidas para fortalecer las posiciones militares estadounidenses en América Latina.

El Estado está llamado a desempeñar un papel clave en el proceso de reordenamiento global. El desprestigio del mercado, que afecta de manera especial al capital financiero en sentido restringido, contribuye a legitimar esta nueva función del Estado. Pero, desde que no basta una redefinición puramente keynesiana del papel de Estado, cabe esperar que se acentúe el autoritarismo, en especial respecto a lograr niveles de gobernabilidad en un contexto de descontento social y criminalidad alimentados por un incremento de la pobreza y el desempleo. 


\section{Bibliografía}

Bureau of Economic Analysis (BEA), Tabla 1.1.1, 2009a, http://www.bea.gov/national/ nipaweb/SelectTable.aps?Selected $=Y(a c-$ tualizado el 15 de junio). , 2009b, Tabla 1.15, http://www. bea.gov/national/nipaweb/TablePrint. asp?Selected $=Y$

Internacional Transactions Accounts Data, Tabla I, 2009c, http://www.bea.gov/ international/xls/table1.xls (actualizado el 14 de septiembre).

Bureau of Labor Statistics (BLS), Database, 2009, http://data.bls.gov/PDQ/servlet/ SurveyOutputServlet?series_id=14000000 (actualizado el 27 de septiembre).

Buchele, Robert y Jens Christiansen, Globalization and the Declining Share of Labor Income in the United States, junio de 2007, http://gesd.free.fr/paper419.pdf (última consulta, 13 de agosto de 2009).

Comisión para la Cooperación Laboral (CCL), Washington, DC, 2003, http://www.naalc. org/spanish/pdf/labor_markets_sp_2.pdf (última consulta, 12 de septiembre 2009).

Dumenil, Gerard y Dominique Lévy, The Real and Financial Components of Profitability (USA 1948-2000), 2004, http://www.jourdon.ens.fr/levy/dle2004g.pdf (última consulta, 3 de septiembre de 2009).

Economic Report of the President, 2007, http:// www.gpoaccess.gov/eop/2007/2007_erp. pdf (última consulta, 3 de agosto de 2009).

Figueroa Sepúlveda, Víctor, "La gestión estatal de la crisis en Estados Unidos", Vínculo jurídico, Zacatecas, Universidad Autónoma de Zacatecas, núm. 11-12, julio-diciembre de 1992, pp. 37-49, y núm. 13, enero-marzo de 1993, pp. 10-21.

Grupo Intergubernamental sobre Cambio Climático (IPCC), Cambio climático 2007: Informe de Síntesis, Contribución de los grupos de trabajo I, II y III al IV Informe de Evaluación del Grupo Intergubernamental de Expertos sobre el Cambio Climático [equipo de redacción principal: Pachauri, R.K. y A. Reisinger, dirs.], Ginebra, IPCC, 2007.

Houseman, Susan, Outsourcing, Offshoring, and Productivity Measurement in US Manufacturing, W. E. Upjohn Institute for Employment Research, 2006, http://www. upjohninst.org/publications/wp/06-130.pdf (consultado en abril de 2007).
Jackson, James K, "Financing the US trade deficit", Congressional Research Service, 2008, http://fpc.state.gov/documents/organization/105196.pdf (actualizado el 12 de abril, última consulta, 14 de junio de 2009).

Lynch, Lisa M., "Organizational innovation and US productivity", VOX, Research-based policy analisis and commentary from leading economists, 6 de diciembre de 2007, http:// www.voxeu.org/index.php?q=node/775) (última consulta, 23 de agosto de 2009).

OECD, Stat Extracts. Labour productivity Growth, 2009, http://stats.oecd.org/Index. aspx?DataSetCode=PDYGTH (actualizado el 11 de septiembre, última consulta, 14 de septiembre).

Palley, Thomas I., "Financialization: What it is and why it matters", Working Paper núm. 525, Washington DC, The Levy Economics Institute and Economics For Democratic and Open Societies, diciembre de 2007, http://www.levy.org/pubs/wp_525.pdf (última consulta, 28 de septiembre de 2009).

Red de Indicadores de Ciencia y Tecnología (RICYT), 2009, http://www.ricyt.org/interior/ interior.asp? Nivel1 $=1 \&$ Nivel2 $=1 \&$ Idioma= (última consulta, 4 de septiembre).

Stern, Nicholas, Stern Review on the Economics of Climate Change, H.M. Treasury, U.K., 2006, http://www.hm-treasury.gov. uk/stern_review_report.htm (última consulta, 25 de septiembre de 2009).

Stiroh, Kevin, Information Technology and the U.S. Productivity Revival: A Review of the Evidence, 2002, http://www2.newyorkfed. $\mathrm{org} / \mathrm{research} /$ economists/stiroh/ks_busec. pdf (última consulta, 13 de junio de 2009).

UNCTAD, "The global economic crisis: systemic failures and multilateral remedies", Report by the UNCTAD Secretariat Task Force on Systemic Issues on Economic Cooperation, United Nations Publications, UNCTAD/GDS/2009/1, 2009, http://www. unctad.org/en/docs/gds20091_en.pdf (última consulta, 26 de julio de 2009).

US Census Bureau, Housing and Houshold Economic Division. Historical Income Tables-Families, 2009, http://www.census. gov/hhes/www/income/histinc/f02AR. html (última actualización, 28 de agosto). 